Tohoku Math. J.

55 (2003), 255-269

\title{
LONG TIME BEHAVIOR OF THE TRANSITION PROBABILITY OF A RANDOM WALK WITH DRIFT ON AN ABELIAN COVERING GRAPH
}

\author{
TOMOYUKI SHIRAI
}

(Received May 16, 2001, revised June 26, 2002)

\begin{abstract}
For a certain class of reversible random walks possibly with drift on an abelian covering graph of a finite graph, using the technique of twisted transition operator, we obtain the asymptotic behavior of the $n$-step transition probability $p_{n}(x, y)$ as $n \rightarrow \infty$ and give an expression of the constant which appears in the asymptotics.
\end{abstract}

1. Introduction. A random walk on $\boldsymbol{Z}^{d}$ defined by the sum of independent $\boldsymbol{Z}^{d}$-valued random variables has been intensively investigated by many authors (cf. $[9,10])$. From the definition it is a Markov chain that is homogeneous in time and space. A Markov chain which has weaker spatial homogeneity than the above is called a semi-Markov chain or sometimes a random walk with internal states (internal degrees of freedom). The asymptotic behavior of the $n$-step transition probability or central limit theorems for semi-Markov chains have been studied under mild conditions (cf. [3, 4, 8]. In a previous paper [5], we obtain an expression of the constant which appears in the asymptotic behavior of the $n$-step transition probabilities for a certain class of semi-Markov chains in terms of geometric quantity, namely, the volume of a Jacobian torus $[1,5,6,7]$. In the present paper, we extend the result obtained in [5] to a wider class of symmetric random walks possibly with drift.

Let $G=(V(G), E(G))$ be an infinite, connected and locally finite graph, where $V(G)$ is the vertex set of $G$ and $E(G)$ is the oriented edges of $G$. For an edge $e \in E(G), o(e)$ (resp. $t(e)$ ) is the origin of $e$ (resp. the terminus of $e$ ) and $\bar{e}$ is the inverse edge of $e$. We assume that $G$ has an abelian group $\Gamma$ of automorphisms which acts on $G$ freely, that is, $\sigma x \neq x, \sigma e \neq \bar{e}$ for every $\sigma(\neq 1) \in \Gamma$, and the quotient graph $M=\Gamma \backslash G$ is finite. We can regard $G$ as the abelian covering graph of $M$ with covering transformation group $\Gamma$ and denote its covering map by $\pi: G \rightarrow M$.

We consider a transition probability $p: E(G) \rightarrow(0,1]$ satisfying the following conditions:

(1) $\sum_{e \in E_{x}(G)} p(e)=1$ for any $x \in V(G)$, where $E_{x}(G)=\{e \in E(G) \mid o(e)=x\}$,

(2) $p$ is invariant under the $\Gamma$-action, that is, $p(\sigma e)=p(e)$ for any $\sigma \in \Gamma$ and $e \in$ $E(G)$

2000 Mathematics Subject Classification. Primary 60J10; Secondary 60G50.

This work is partially supported by JSPS under the Grant-in-Aid for Scientific Research No. 13740057. 
(3) $\quad p$ is $m$-symmetric, that is, there exists a positive function $m: V(G) \rightarrow(0, \infty)$ such that

$$
m(o(e)) p(e)=m(t(e)) p(\bar{e}) .
$$

The function $m$ is called the reversible measure for $p$. If a reversible measure exists, it is unique up to a constant multiple. We define the transition operator $P$ by

$$
P f(x)=\sum_{e \in E_{x}(G)} p(e) f(t(e))
$$

which, by (1.1), turns out to be a self-adjoint operator on $\ell^{2}(V(G))$ equipped with the inner product $\langle f, g\rangle=\sum_{x \in V(G)} f(x) \overline{g(x)} m(x)$.

Our main concern is the asymptotic behavior of the $n$-step transition probability defined by

$$
p_{n}(x, y)=\sum_{\substack{|c|=n \\ \mid\left(e_{1}\right)=x, t\left(e_{n}\right)=y}} p(c),
$$

where a path $c=\left(e_{1}, e_{2}, \ldots, e_{n}\right)$ of length $n$ is a sequence of $n$ edges such that $t\left(e_{i}\right)=$ $o\left(e_{i+1}\right)$ for $1 \leq i \leq n-1,|c|(=n)$ is the length of $c$ and $p(c)=\prod_{i=1}^{n} p\left(e_{i}\right)$.

In a previous paper [5], we assumed that $m$ is invariant under the $\Gamma$-action, so that we necessarily dealt with "isotropic" random walks (see Proposition 3.1 and Remark 3.2 below). In this paper, we do not assume the $\Gamma$-invariance of $m$, that is, the existence of a reversible measure on the quotient graph $M$.

Our setting includes a certain class of non-isotropic reversible random walks, for example, the random walk on one-dimensional lattice $Z^{1}$ whose transition probability is given by

$$
p(x, x+1)=p, \quad p(x, x-1)=q \quad \text { for any } x \in Z^{1},
$$

where $p+q=1$ with $p, q>0$. In this case, we may take $M$ to be the 1-bouquet graph with $\Gamma=Z^{1}$, and the reversible measure is given by $m(x)=(p / q)^{x}$. However, $m$ is not $\Gamma$-invariant unless $p=q=1 / 2$. It is easy to see that

$$
p_{2 n}(x, x) \sim \frac{1}{\sqrt{\pi n}}(2 \sqrt{p q})^{2 n}
$$

as $n \rightarrow \infty$.

Let $Q=(q(x, y))_{x, y \in V(M)}$ be the finite symmetric matrix on $M$ whose elements are defined by

$$
q(x, y)=\sum_{o(e)=x, t(e)=y} q(e)
$$

where

$$
q(e)=(p(e) p(\bar{e}))^{1 / 2}
$$

for each $e \in E(M)$. In our setting, the matrix $Q$ plays an important role and the asymptotic behavior of the transition probability $p_{n}(x, y)$ is controlled by it. The quantity $2 \sqrt{p q}$ 
which appeared in (1.5) is closely related to the above symmetrization of the transition probability $p$.

We will prove the following result:

THEOREM 1.1. (1) The maximum of the spectrum of $P$ on $\ell^{2}(V(G))$, say $\lambda_{1}:=$ $\max \operatorname{Spec}(P)$, is equal to the maximal eigenvalue of $Q$.

(2) Let $\hat{\Gamma}$ be the group of unitary characters of $\Gamma$ and $k=\operatorname{rank} \Gamma$.

(i) When $G$ is non-bipartite, we have

$$
p_{n}(x, y) \sim \frac{\exp \left(\frac{1}{2} \int_{x}^{y} d \log m\right) \varphi_{0}(\pi(x)) \varphi_{0}(\pi(y))}{\operatorname{Vol}_{q}(\hat{\Gamma})} \frac{\lambda_{1}^{n+(k / 2)}}{(4 \pi n)^{k / 2}}
$$

as $n \rightarrow \infty$.

(ii) When $G$ is bipartite with bipartition $V(G)=A \sqcup B$, and

(a) if $x, y \in A$ or $x, y \in B$, for even $n \rightarrow \infty$, or (b) if $x \in A, y \in B$ or $x \in B, y \in A$, for odd $n \rightarrow \infty$, we have

$$
p_{n}(x, y) \sim \frac{2 \exp \left(\frac{1}{2} \int_{x}^{y} d \log m\right) \varphi_{0}(\pi(x)) \varphi_{0}(\pi(y))}{\operatorname{Vol}_{q}(\hat{\Gamma})} \frac{\lambda_{1}^{n+(k / 2)}}{(4 \pi n)^{k / 2}} .
$$

In the theorem, the quantity $\exp \left(1 / 2 \int_{x}^{y} d \log m\right)$ is equal to $\sqrt{m(y) / m(x)}$. The coboundary operator $d$ and the integral $\int_{x}^{y}$ are defined in Section 2. The function $\varphi_{0}$ is the (positive) normalized eigenfunction for the maximal eigenvalue of $Q$, that is,

$$
Q \varphi_{0}=\lambda_{1} \varphi_{0}, \quad\left\|\varphi_{0}\right\|_{0}^{2}=\sum_{x \in V(M)}\left|\varphi_{0}(x)\right|^{2}=1 .
$$

The 1-cohomology group $H^{1}(M, \boldsymbol{R})$ can be identified with the space of harmonic 1-forms on $M$ by introducing the inner product

$$
\left\langle\left\langle\omega_{1}, \omega_{2}\right\rangle\right\rangle_{q}=\frac{1}{2} \sum_{e \in E(M)} \omega_{1}(e) \omega_{2}(e) q(e) \varphi_{0}(o(e)) \varphi_{0}(t(e))
$$

on $C^{1}(M, \boldsymbol{R})$, the space of 1 -forms on $M$. The group $\hat{\Gamma}$ is identified with a subset of the Jacobian torus $J(M)=H^{1}(M, \boldsymbol{R}) / H^{1}(M, \boldsymbol{Z})$ by the canonical injection. The volume $\operatorname{Vol}_{q}(\hat{\Gamma})$ is that of $\hat{\Gamma}$ with respect to the Lebesgue measure associated with the inner product $\langle\cdot \cdot, \cdot\rangle_{q}$.

REMARK 1.2. By Proposition 3.1, $\lambda_{1}=1$ if and only if $m$ is $\Gamma$-invariant, and then the positive normalized eigenfunction is $\varphi_{0}(x)=(m(x) / m(\mathcal{F}))^{1 / 2}$, where $\mathcal{F}$ is a fundamental set of $G$ for $\Gamma$ and $m(\mathcal{F})=\sum_{x \in \mathcal{F}} m(x)$. It is easy to check that if $m$ is $\Gamma$-invariant and $G$ is non-bipartite it holds that

$$
p_{n}(x, y) \sim \frac{m(y) m(\mathcal{F})^{k / 2-1}}{\operatorname{Vol}(\hat{\Gamma})}(4 \pi n)^{-k / 2}
$$

as $n \rightarrow \infty$, which has been obtained in [5]. 
Our method is also applied to continuous time random walks. Let $p_{t}(x, y)$ is the transition probability of the continuous time random walk with generator $\Delta=P-I$. Then we have the following.

THEOREM 1.3. Let $\hat{\Gamma}$ be the unitary character of $\Gamma$ and $k=\operatorname{rank} \Gamma$. Then we have

$$
p_{t}(x, y) \sim \frac{\exp \left(\frac{1}{2} \int_{x}^{y} d \log m\right) \varphi_{0}(\pi(x)) \varphi_{0}(\pi(y))}{\operatorname{Vol}_{q}(\hat{\Gamma})} \times \frac{e^{-t \alpha_{1}}}{(4 \pi t)^{k / 2}}
$$

as $t \rightarrow \infty$, where $\alpha_{1}\left(=1-\lambda_{1}\right)$ is the minimal eigenvalue of $-\Delta$.

2. The twisted transition operators. We let

$$
\begin{aligned}
& C^{0}(M, A)=\{f: V(M) \rightarrow A\}, \\
& C^{1}(M, A)=\{\omega: E(M) \rightarrow A \mid \omega(\bar{e})=-\omega(e)\},
\end{aligned}
$$

where $A$ is an abelian group. Define the coboundary operator $d: C^{0}(M, A) \rightarrow C^{1}(M, A)$ by

$$
d f(e)=f(t(e))-f(o(e)),
$$

and consider the 1-cohomology group

$$
H^{1}(M, \boldsymbol{R})=C^{1}(M, \boldsymbol{R}) / \operatorname{Image}(d) .
$$

We take inner products $\langle\cdot, \cdot\rangle$ on $C^{0}(M, \boldsymbol{R})$ and $\langle\langle\cdot, \cdot\rangle\rangle$ on $C^{1}(M, \boldsymbol{R})$. With respect to them, we obtain the adjoint operator $\delta$ of $d$ satisfying $\langle\langle d f, \omega\rangle\rangle=\langle f, \delta \omega\rangle$. We say that $\omega \in C^{1}(M, \boldsymbol{R})$ is a harmonic 1 -form if $\delta \omega=0$. So one can identify $H^{1}(M, \boldsymbol{R})$ with the space of harmonic 1 -forms, that is,

$$
H^{1}(M, \boldsymbol{R}) \cong\left\{\omega \in C^{1}(M, \boldsymbol{R}) \mid \delta \omega=0\right\}
$$

and under this identification

$$
H^{1}(M, Z) \cong\left\{\omega \in H^{1}(M, \boldsymbol{R}) \mid \int_{c} \omega \in \boldsymbol{Z} \text { for any closed path } c \text { in } M\right\},
$$

where

$$
\int_{c} \omega=\sum_{i=1}^{n} \omega\left(e_{i}\right)
$$

for a path $c=\left(e_{1}, e_{2}, \ldots, e_{n}\right)$. In the next section, in order to compute the Hessian of the maximal eigenvalue of a twisted transition operator, we will choose appropriate inner products on $C^{0}(M, \boldsymbol{R})$ and $C^{1}(M, \boldsymbol{R})$.

Let $H_{1}(M, Z)$ be the 1-homology group of $M$ and $\hat{H}_{1}(M, Z)$ the unitary character of $H_{1}(M, Z)$. We identify $\hat{H}_{1}(M, Z)$ with the Jacobian torus $J(M)=H^{1}(M, \boldsymbol{R}) / H^{1}(M, Z)$ by the mapping

$$
H^{1}(M, \boldsymbol{R}) \ni \omega \mapsto \chi_{\omega} \in \hat{H}_{1}(M, \boldsymbol{Z}),
$$


where

$$
\chi_{\omega}(\sigma)=\exp \left(2 \pi \sqrt{-1} \int_{c} \omega\right)
$$

and $\sigma$ is represented by 1 -cycle $c$ in $M$.

There exists an abelian covering graph $M^{a b}$ of $M$ whose covering transformation group is $H_{1}(M, Z)$. The graph $M^{a b}$ is called the maximal abelian covering graph. For any covering graph $G$ of $M$, whose covering transformation group $\Gamma$, there exists a covering map $M^{a b} \rightarrow$ $G$ which factorizes the covering map $M^{a b} \rightarrow M$. We remark that if the transformation group of the covering map $M^{a b} \rightarrow G$ is $\Gamma_{1}$, then $\Gamma$ is isomorphic to $H_{1}(M, Z) / \Gamma_{1}$.

Let $\hat{\Gamma}$ be the group of unitary characters of $\Gamma$. The surjective homomorphism $\eta$ : $H_{1}(M, \boldsymbol{Z}) \rightarrow \Gamma \cong H_{1}(M, \boldsymbol{Z}) / \Gamma_{1}$ induces the injective homomorphism $\hat{\eta}: \hat{\Gamma} \rightarrow \hat{H}_{1}(M, \boldsymbol{Z}) \cong$ $J(M)$, and so $\hat{\Gamma}$ is identified with the image in $J(M)$ by $\hat{\eta}$. Then $\chi_{\omega} \in \hat{\Gamma}$ if and only if $\int_{c} \omega \in \boldsymbol{Z}$ for every 1 -cycle in $M$ with $\eta(c)=1$. It is easy to check that the tangent space $T_{\mathbf{1}} \hat{\Gamma}$ of $\hat{\Gamma}$ at the trivial character $\mathbf{1}$ coincides with

$$
\left\{\omega \in H^{1}(M, \boldsymbol{R}) \mid \int_{c} \omega=0 \text { for every 1-cycle } c \text { in } M \text { with } \eta(c)=1\right\} .
$$

We have the Euclidean metric $\langle\cdot, \cdot\rangle\rangle$ on $H^{1}(M, \boldsymbol{R})$ and induce it on $J(M)$ by $\hat{\eta}$. The volume is measured with respect to this metric and denoted by $\operatorname{Vol}(\hat{\Gamma})$.

In what follows, we choose an arbitrary vertex $x_{0} \in V(G)$ and fix it. Let $\tilde{\omega}$ be the lift of $\omega \in T_{\mathbf{1}} \hat{\Gamma}$ to $E(G)$. We define a function $s$ on $V(G)$ by

$$
s(x)=s_{\chi_{\omega}}(x)=\exp \left(2 \pi \sqrt{-1} \int_{x_{0}}^{x} \tilde{\omega}\right),
$$

where $\int_{x_{0}}^{x} \tilde{\omega}=\sum_{i=1}^{n} \omega\left(e_{i}\right)$ for a path $x_{0} x=\left(e_{1}, e_{2}, \ldots, e_{n}\right)$. The function $s$ is independent of the choice of a path joining $x_{0}$ to $x$ and

$$
s(\sigma x)=\chi_{\omega}(\sigma) s(x)
$$

for any $\sigma \in \Gamma$.

Next, we define the function $\ell$ on $V(G) \times V(G)$ by

$$
\ell(x, y)=\prod_{i=1}^{n} \frac{p\left(e_{i}\right)}{p\left(\bar{e}_{i}\right)} \in(0, \infty)
$$

where $x y=\left(e_{1}, e_{2}, \ldots, e_{n}\right)$. Since $p$ is $m$-symmetric, we have

$$
\ell(x, y)=\prod_{i=1}^{n} \frac{m\left(t\left(e_{i}\right)\right)}{m\left(o\left(e_{i}\right)\right)}=\frac{m\left(t\left(e_{n}\right)\right)}{m\left(o\left(e_{1}\right)\right)}=\frac{m(y)}{m(x)},
$$

and so it is independent of the choice of a path $\left(e_{1}, e_{2}, \ldots, e_{n}\right)$ joining $x$ to $y$. We note that $\ell(x, y)$ can be represented by

$$
\ell(x, y)=\exp \left(\int_{x}^{y} d \log m\right),
$$

where $d$ is the one defined by (2.2). 
LEMMA 2.1. (1) Let $\ell$ be defined as above. Then we have

$$
\ell(x, x)=1, \quad \ell(y, x)=\ell(x, y)^{-1}, \quad m(y)=m(x) \ell(x, y) .
$$

(2) $\ell(x, \sigma x)$ is independent of $x \in V(G)$ and so

$$
\ell(\sigma):=\ell(x, \sigma x)
$$

is well-defined and $\ell: \Gamma \rightarrow(0, \infty)$ is a multiplicative homomorphism, that is, $\ell(\sigma \eta)=$ $\ell(\sigma) \ell(\eta)$.

PROOF. (1) It is trivial.

(2) Since

$$
\ell(x, \sigma x) \ell(\sigma x, \sigma y) \ell(\sigma y, y) \ell(y, x)=\ell(x, x)=1
$$

and $p$ is $\Gamma$-invariant, we obtain

$$
\frac{\ell(x, \sigma x)}{\ell(y, \sigma y)}=\frac{\ell(x, y)}{\ell(\sigma x, \sigma y)}=\frac{\prod_{i=1}^{n} p\left(e_{i}\right) / p\left(\bar{e}_{i}\right)}{\prod_{i=1}^{n} p\left(\sigma e_{i}\right) / p\left(\sigma \bar{e}_{i}\right)}=1
$$

for a path $x y=\left(e_{1}, e_{2}, \ldots, e_{n}\right)$. The last assertion is obvious.

Let $\pi: G \rightarrow M$ be the canonical projection. We define the weighted lift $\tilde{f}$ of $f \in$ $C^{0}(M, C)$ to $V(G)$ by

$$
\tilde{f}(x)=\alpha\left(x_{0}, x\right) f(\pi(x)),
$$

where $\alpha(x, y)=\ell(x, y)^{-1 / 2}$. We remark that for every $\sigma \in \Gamma$

$$
\tilde{f}(\sigma x)=\alpha(\sigma) \tilde{f}(x)
$$

where $\alpha(\sigma)=\ell(\sigma)^{-1 / 2}$. Let

$$
\ell_{\chi, \alpha}^{2}=\{f: V(G) \rightarrow C \mid f(\sigma x)=\chi(\sigma) \alpha(\sigma) f(x) \text { for } \sigma \in \Gamma\},
$$

which is the finite dimensional $\ell^{2}$-space equipped with the inner product

$$
\langle f, g\rangle_{\chi}=\sum_{x \in \mathcal{F}} f(x) \overline{g(x)} \ell\left(x_{0}, x\right),
$$

where $\mathcal{F}$ is a fundamental set of $G$. The inner product is independent of the choice of a fundamental set $\mathcal{F}$, since

$$
\begin{aligned}
f(\sigma x) \overline{g(\sigma x)} \ell\left(x_{0}, \sigma x\right) & =\chi(\sigma) \alpha(\sigma) f(x) \overline{\chi(\sigma) \alpha(\sigma) g(x)} \ell\left(x_{0}, x\right) \ell(\sigma) \\
& =f(x) \overline{g(x)} \ell\left(x_{0}, x\right) .
\end{aligned}
$$

Let $\ell^{2}(V(M))$ be the $\ell^{2}$-space of complex-valued functions on $V(M)$ with the inner product

$$
\langle f, g\rangle_{0}=\sum_{x \in V(M)} f(x) \overline{g(x)} .
$$


It is immediate to see that $\tilde{f} s \in \ell_{\chi, \alpha}^{2}$ from (2.11) and (2.20). Then we define the operator $U: \ell^{2}(V(M)) \rightarrow \ell_{x, \alpha}^{2}$ by $U f=\tilde{f} s$.

LEMmA 2.2. The operator $U:\left(\ell^{2}(V(M)),\langle\cdot, \cdot\rangle_{0}\right) \rightarrow\left(\ell_{\chi, \alpha}^{2},\langle\cdot, \cdot\rangle_{\chi}\right)$ is unitary.

PRoOF. Using (2.19) and Lemma 2.1 (1), we obtain

$$
\begin{aligned}
\langle U f, U g\rangle_{\chi} & =\sum_{x \in \mathcal{F}} \tilde{f}(x) s(x) \overline{\tilde{g}(x) s(x)} \ell\left(x_{0}, x\right) \\
& =\sum_{x \in \mathcal{F}} \alpha\left(x_{0}, x\right) f(\pi(x)) \overline{\alpha\left(x_{0}, x\right) g(\pi(x))} \ell\left(x_{0}, x\right) \\
& =\sum_{x \in V(M)} f(x) \overline{g(x)}=\langle f, g\rangle_{0} .
\end{aligned}
$$

Since the dimensions of $\ell^{2}(V(M))$ and $\ell_{\chi, \alpha}^{2}$ are the same, the operator $U$ is unitary.

It is easy to check that $P$ leaves $\ell_{\chi, \alpha}^{2}$ invariant, that is, $P\left(\ell_{\chi, \alpha}^{2}\right) \subset \ell_{\chi, \alpha}^{2}$. The restriction $P_{\chi}=\left.P\right|_{\ell_{\chi, \alpha}^{2}}$ is called the twisted transition operator. Put $L_{\chi}=U^{-1} P_{\chi} U: \ell^{2}(V(M)) \rightarrow$ $\ell^{2}(V(M))$. Then we find the concrete form of $L_{\chi}$.

LEMMA 2.3. Let $q(e)=(p(e) p(\bar{e}))^{1 / 2}$ for each $e \in E(M)$. Then,

$$
L_{\chi} f(x)=\sum_{e \in E_{x}(M)} q(e) \exp (2 \pi \sqrt{-1} \omega(e)) f(t(e)) .
$$

Proof. From the definition of $U$, we have

$$
\begin{aligned}
P_{\chi} U f(x) & =\sum_{e \in E_{x}(G)} p(e) \alpha\left(x_{0}, t(e)\right) f(\pi(t(e))) s(t(e)) \\
& =\sum_{e \in E_{x}(G)} p(e)\left(\frac{p(e)}{p(\bar{e})}\right)^{-1 / 2} \alpha\left(x_{0}, x\right) f(\pi(t(e))) \exp (2 \pi \sqrt{-1} \omega(e)) s(x) \\
& =\left(\sum_{e \in E_{\pi(x)}(M)} q(e) \exp (2 \pi \sqrt{-1} \omega(e)) f(t(e))\right) \alpha\left(x_{0}, x\right) s(x) .
\end{aligned}
$$

Hence we obtain the lemma.

We enumerate the eigenvalues of $L_{\chi}$ (or $P_{\chi}$ ) by

$$
\lambda_{1}(\chi) \geq \lambda_{2}(\chi) \geq \cdots \geq \lambda_{N}(\chi),
$$

where $N=|\mathcal{F}|$. By the theory of direct integral we obtain

$$
\operatorname{Spec}(P)=\bigcup_{i=1}^{N} \bigcup_{\chi \in \hat{\Gamma}}\left\{\lambda_{i}(\chi)\right\} .
$$

We remark that $L_{\mathbf{1}}$ is equal to the finite matrix $Q$ defined in (1.6) and may not be a stochastic matrix. 
3. Properties of $\lambda_{1}(\chi)$. In this section we show some properties of $\lambda_{1}(\chi)$. In the proofs we will use three kinds of (modified) coboundary operators and their duals with respect to the pairs of inner products in (3.5), (3.13) and (3.21).

The asymptotic behavior of the transition probability is determined by the behavior of the largest eigenvalue $\lambda_{1}(\chi)$ of $L_{\chi}$ around the trivial character 1. First we remark the relationship between $\lambda_{1}(\mathbf{1})$ and the existence of a reversible measure for $p$ on the quotient graph $M$.

Proposition 3.1. Assume that there exists a reversible measure $m$ on $V(G)$ for a transition probability $p: E(G) \rightarrow(0,1]$ which is $\Gamma$-invariant. Then the following three conditions are equivalent:

(1) The reversible measure $m$ is $\Gamma$-invariant.

(2) There exists a reversible measure $m_{0}$ on $V(M)$.

(3) $\lambda_{1}(\mathbf{1})=1$.

Proof. It is obvious that (1) and (2) are equivalent, so that it suffices to show (2) and (3) are equivalent. Assume that there exists a reversible measure $m_{0}$ on $V(M)$. Since $m_{0}(o(e)) p(e)=m_{0}(t(e)) p(\bar{e})$, we find that

$$
m_{0}(o(e)) p(e)^{2}=m_{0}(t(e)) p(\bar{e}) p(e)=m_{0}(t(e)) q(e)^{2} .
$$

Then we have

$$
\left(L_{\mathbf{1}} m_{0}^{1 / 2}\right)(x)=\sum_{e \in E_{x}(M)} q(e) m_{0}^{1 / 2}(t(e))=\sum_{e \in E_{x}(M)} p(e) m_{0}^{1 / 2}(o(e))=m_{0}^{1 / 2}(x)
$$

and so $\lambda_{1}(\mathbf{1})=1$.

Conversely, we assume that $L_{\mathbf{1}}$ has the eigenvalue 1, and let $\varphi$ be its corresponding eigenfunction, that is, $L_{\mathbf{1}} \varphi=\varphi$. We define the modified coboundary operator $D: C^{0}(M, \boldsymbol{R}) \rightarrow$ $C^{1}(M, \boldsymbol{R})$ by

$$
D f(e)=p(\bar{e})^{1 / 2} f(t(e))-p(e)^{1 / 2} f(o(e))
$$

and define $D^{*}: C^{1}(M, \boldsymbol{R}) \rightarrow C^{0}(M, \boldsymbol{R})$ by

$$
D^{*} \omega(x)=-\sum_{e \in E_{x}(M)} p(e)^{1 / 2} \omega(e) .
$$

It is easy to check that $D$ and $D^{*}$ are mutually adjoint operators with respect to the inner products

$$
\langle f, g\rangle_{0}=\sum_{x \in V(M)} f(x) g(x), \quad\left\langle\langle\omega, \eta\rangle_{0}=\frac{1}{2} \sum_{e \in E(M)} \omega(e) \eta(e)\right.
$$

and that

$$
D^{*} D f(x)=f(x)-\sum_{e \in E_{x}(M)} q(e) f(t(e))=\left(I-L_{\mathbf{1}}\right) f(x) .
$$

For the eigenfunction $\varphi$ as above, since

$$
\left\langle\langle D \varphi, D \varphi\rangle_{0}=\left\langle D^{*} D \varphi, \varphi\right\rangle_{0}=\left\langle\left(I-L_{\mathbf{1}}\right) \varphi, \varphi\right\rangle_{0}=0,\right.
$$


we get

$$
D \varphi(e)=p(\bar{e})^{1 / 2} \varphi(t(e))-p(e)^{1 / 2} \varphi(o(e))=0
$$

for any $e \in E(M)$, and this implies that $\varphi(x)^{2}$ is a reversible measure for $p$ on $V(M)$.

REMARK 3.2. We assumed the existence of a reversible measure on $V(G)$ but not the $\Gamma$-invariance of the reversible measure on $V(G)$. Since the $\Gamma$-invariance of the reversible measure on $V(G)$ implies that $\lambda_{1}(\mathbf{1})=1$, the corresponding random walk is "isotropic".

The eigenvalue $\lambda_{1}(\mathbf{1})$ is the largest one among the first eigenvalues of $L_{\chi}$. Since we consider only abelian covering graphs, $\lambda_{1}(\mathbf{1})$ coincides with the maximum of the spectrum of $P$.

PROPOSITION 3.3. For any unitary character $\chi, \lambda_{1}(\chi) \leq \lambda_{1}(\mathbf{1})$. The equality holds if and only if $\chi=1$. In particular, the maximum of $\operatorname{Spec}(P)$ is given by the maximal eigenvalue of the finite matrix $Q$ defined in (1.6).

Proof. The last assertion is obtained from the first assertion and (2.29).

For a 1-form $\theta \in C^{1}(M, \boldsymbol{R})$ such that $\chi=\chi_{\theta}$, put

$$
C_{\theta}^{1}(M, C)=\{\omega: E(M) \rightarrow C \mid \omega(\bar{e})=-\exp (-2 \pi \sqrt{-1} \theta(e)) \omega(e) \text { for } e \in E(M)\} .
$$

We define the modified coboundary operator $D_{\theta}: C^{0}(M, C) \rightarrow C_{\theta}^{1}(M, C)$ by

$$
D_{\theta} f(e)=\exp (2 \pi \sqrt{-1} \theta(e)) \frac{f(t(e))}{\varphi_{0}(t(e))}-\frac{f(o(e))}{\varphi_{0}(o(e))},
$$

where $\varphi_{0}$ is the positive solution to the equation

$$
L_{\mathbf{1}} \varphi_{0}=\lambda_{1}(\mathbf{1}) \varphi_{0}
$$

with $\left\|\varphi_{0}\right\|_{0}=1$. Also, we define $D_{\theta}^{*}: C_{\theta}^{1}(M, C) \rightarrow C^{0}(M, C)$ by

$$
D_{\theta}^{*} \omega(x)=-\sum_{e \in E_{x}(M)} \omega(e) q(e) \varphi_{0}(t(e)) .
$$

It is easy to check that $D_{\theta}$ and $D_{\theta}^{*}$ are mutually adjoint operators with respect to the inner products

$$
\begin{aligned}
\langle f, g\rangle_{0} & =\sum_{x \in V(M)} f(x) \overline{g(x)}, \\
\left\langle\langle\omega, \eta\rangle_{q}\right. & =\frac{1}{2} \sum_{e \in E(M)} \omega(e) \overline{\eta(e)} q(e) \varphi_{0}(o(e)) \varphi_{0}(t(e)),
\end{aligned}
$$

and moreover we see that

$$
\begin{aligned}
D_{\theta}^{*} D_{\theta} f(x) & =\lambda_{1}(\mathbf{1}) f(x)-\sum_{e \in E_{x}(M)} q(e) \exp (2 \pi \sqrt{-1} \theta(e)) f(t(e)) \\
& =\left(\lambda_{1}(\mathbf{1}) I-L_{\chi_{\theta}}\right) f(x) .
\end{aligned}
$$


Hence we obtain

$$
\left\langle\left\langle D_{\theta} f, D_{\theta} f\right\rangle_{q}=\left\langle D_{\theta}^{*} D_{\theta} f, f\right\rangle_{0}=\left\langle\left(\lambda_{1}(\mathbf{1}) I-L_{\chi_{\theta}}\right) f, f\right\rangle_{0} \geq 0\right.
$$

First assertion immediately follows from (3.15).

Assume that $L_{\chi_{\theta}} f=\lambda_{1}(\mathbf{1}) f$ and $f \neq 0$. Then, by (3.15), we get $D_{\theta} f=0$, or equivalently,

$$
\exp (2 \pi \sqrt{-1} \theta(e)) \frac{f(t(e))}{\varphi_{0}(t(e))}=\frac{f(o(e))}{\varphi_{0}(o(e))}
$$

for any $e \in E(M)$. Since $M$ is connected, $f(x)$ is non-zero for any $x \in V(M)$. Thus for any closed path $c$ in $M$, we have

$$
\exp \left(2 \pi \sqrt{-1} \int_{c} \theta\right)=1
$$

which implies $\chi=\mathbf{1}$.

The infimum of the spectrum of $P$ is closely related to the bipartiteness of a graph $G$. By using the technique of the proof above we can show the following proposition.

Proposition 3.4. The spectrum of $P$ is contained in the closed interval $\left[-\lambda_{1}(\mathbf{1}), \lambda_{1}(\mathbf{1})\right]$. Furthermore, $\inf \operatorname{Spec}(P)=-\lambda_{1}(\mathbf{1})$ if and only if $G$ is bipartite.

Proof. Take a 1 -form $\theta_{1 / 2} \in C^{1}(M, \boldsymbol{R})$ such that $\left|\theta_{1 / 2}\right| \equiv 1 / 2$, and set $\theta^{\prime}=\theta+\theta_{1 / 2}$ for a 1 -form $\theta$. We consider $\theta^{\prime}$ as $\theta$ in the proof of Proposition 3.3. Then we obtain

$$
\left\langle\left\langle D_{\theta^{\prime}} f, D_{\theta^{\prime}} f\right\rangle\right\rangle_{q}=\left\langle\left(\lambda_{1}(\mathbf{1}) I+L_{\chi_{\theta}}\right) f, f\right\rangle_{0} \geq 0 .
$$

This inequality together with (3.15) and (2.29) implies that $\operatorname{Spec}(P) \subset\left[-\lambda_{1}(\mathbf{1}), \lambda_{1}(\mathbf{1})\right]$.

Assume that $\inf \operatorname{Spec}(P)=-\lambda_{1}(\mathbf{1})$. Then there exists a 1-form $\theta$ such that $\chi_{\theta} \in \hat{\Gamma}$ and $L_{\chi_{\theta}} f=-\lambda_{1}(\mathbf{1}) f$, and hence $D_{\theta^{\prime}} f=0$, or equivalently,

$$
-\exp (2 \pi \sqrt{-1} \theta(e)) \frac{f(t(e))}{\varphi_{0}(t(e))}=\frac{f(o(e))}{\varphi_{0}(o(e))}
$$

for any $e \in E(M)$. From this equation, we get

$$
\exp \left(2 \pi \sqrt{-1} \int_{c} \theta\right)= \begin{cases}1, & \text { if }|c| \text { is even, } \\ -1, & \text { if }|c| \text { is odd, }\end{cases}
$$

for any closed path $c$ in $M$, which implies that $\chi_{\theta}(\sigma)= \pm 1$ for any $\sigma \in \Gamma$. Let $\tilde{c}$ be an arbitrary closed path in $G$ and $c$ its projection onto $M$. Then, since $\chi_{\theta} \in \hat{\Gamma}, \chi_{\theta}(c)$ is equal to 1 and hence $|\tilde{c}|$ must be even from (3.20). Consequently, $G$ is bipartite.

The other direction is obvious from Lemma 3.3, since $P$ and $-P$ are unitarily equivalent when $G$ is bipartite. 
For the next proposition we choose inner products

$$
\begin{aligned}
\langle f, g\rangle_{q} & =\sum_{x \in V(M)} f(x) \overline{g(x)} \varphi_{0}(x), \\
\langle\omega \omega, \eta\rangle\rangle_{q} & =\frac{1}{2} \sum_{e \in E(M)} \omega(e) \overline{\eta(e)} q(e) \varphi_{0}(o(e)) \varphi_{0}(t(e)),
\end{aligned}
$$

where $\varphi_{0}$ is the same as in (3.11). We define the operator $\delta_{q}: C^{1}(M, C) \rightarrow C^{0}(M, \boldsymbol{C})$ by

$$
\delta_{q} \omega(x)=-\sum_{e \in E_{x}(M)} \omega(e) q(e) \varphi_{0}(t(e)) .
$$

It is easy to see that the operator $\delta_{q}$ is the dual one of $d$, that is, $\langle d f f, \omega\rangle_{q}=\left\langle f, \delta_{q} \omega\right\rangle_{q}$.

In order to compute the Hessian of $\lambda_{1}(\chi)$ at $\chi=\mathbf{1}$, we take a curve $\chi_{t}=\chi_{t \omega}$ in $\hat{\Gamma}$ such that $\chi_{0}=\mathbf{1}$ and consider the equation

$$
\left\{\begin{array}{l}
L_{t} \varphi_{t}(x)=\lambda_{1}(t) \varphi_{t}(x), \\
\left\|\varphi_{0}\right\|_{0}^{2}=\left\langle\varphi_{0}, 1\right\rangle_{q}=1,
\end{array}\right.
$$

where $L_{t}=L_{\chi_{t}}$ and $\lambda_{1}(t)$ is the maximal eigenvalue of $L_{t}$. In particular,

$$
L_{0} \varphi_{0}(x)=\lambda_{1}(0) \varphi_{0}(x)\left(=\lambda_{1}(\mathbf{1}) \varphi_{0}(x)\right) .
$$

Now we compute the Hessian of $\lambda_{1}$ at the trivial character 1 . Then,

$$
\operatorname{Hess}_{\chi=1} \lambda_{1}(\omega, \omega)=-8 \pi^{2}\|\omega\|_{q}^{2}
$$

Proof. Let $\varphi_{t}$ be defined as in (3.23). Observe that

$$
\begin{aligned}
\left\langle L_{t} \varphi_{t}, 1\right\rangle_{q}= & \sum_{x \in V(M)} \sum_{e \in E_{x}(M)} q(e) \exp (2 \pi \sqrt{-1} t \omega(e)) \varphi_{t}(t(e)) \varphi_{0}(o(e)) \\
= & \sum_{x \in V(M)} \varphi_{t}(x) \sum_{e \in E_{x}(M)} q(e)\left(1-2 \pi \sqrt{-1} t \omega(e)-2 \pi^{2} t^{2} \omega(e)^{2}\right) \varphi_{0}(t(e)) \\
& +O\left(t^{3}\right) \\
= & \sum_{x \in V(M)} \varphi_{t}(x)\left(\lambda_{1}(0) \varphi_{0}(x)+2 \pi \sqrt{-1} t \delta_{q} \omega(x)\right. \\
& \left.-2 \pi^{2} t^{2} \sum_{e \in E_{x}(M)} q(e) \omega(e)^{2} \varphi_{0}(t(e))\right)+O\left(t^{3}\right) \\
= & \lambda_{1}(0)\left\langle\varphi_{t}, 1\right\rangle_{q}-4 \pi^{2} t^{2}\|\omega\|_{q}^{2}+O\left(t^{3}\right)
\end{aligned}
$$

as $t \rightarrow 0$. Here we used the fact that $\omega$ is $q$-harmonic.

Differentiating both sides of the equation

$$
\left\langle L_{t} \varphi_{t}, 1\right\rangle_{q}=\lambda_{1}(t)\left\langle\varphi_{t}, 1\right\rangle_{q}
$$


and putting $t=0$, we obtain

$$
\lambda_{1}(0)\left\langle\varphi_{0}^{\prime}, 1\right\rangle_{q}=\lambda_{1}^{\prime}(0)\left\langle\varphi_{0}, 1\right\rangle_{q}+\lambda_{1}(0)\left\langle\varphi_{0}^{\prime}, 1\right\rangle_{q} .
$$

Then we have $\lambda_{1}^{\prime}(0)=0$.

Differentiating again and putting $t=0$, we obtain

$$
\lambda_{1}(0)\left\langle\varphi_{0}^{\prime \prime}, 1\right\rangle_{q}-8 \pi^{2}\|\omega\|_{q}^{2}=\lambda_{1}^{\prime \prime}(0)\left\langle\varphi_{0}, 1\right\rangle_{q}+\lambda_{1}(0)\left\langle\varphi_{0}^{\prime \prime}, 1\right\rangle_{q} .
$$

Then we have $\lambda_{1}^{\prime \prime}(0)=-8 \pi^{2}\|\omega\|_{q}^{2}$.

4. Proof of the theorems. The assertion of Theorem 1.1 in the case where $G$ is bipartite can be proved in almost the same way as in the case where $G$ is non-bipartite (see [5]). Here we give a proof only for the non-bipartite case. Throughout this section, we assume that $G$ is non-bipartite.

For any $x, y \in V(G)$ we can take a fundamental set $\mathcal{F}$ such that $x, y \in \mathcal{F}$. Let

$$
f_{x, \chi}=\alpha\left(x, x_{0}\right) s_{\chi}(x)^{-1} U \delta_{\pi(x)} \in \ell_{\chi, \alpha}^{2},
$$

where $\delta_{\pi(x)} \in \ell^{2}(V(M))$ is the delta function supported by $\pi(x)$ and $U$ is the one defined in Section 2 . It is easy to check that

$$
f_{x, \chi}(z)=\left\{\begin{array}{cc}
\chi(\sigma) \alpha(\sigma) & \text { if } z=\sigma x \\
0 & \text { otherwise }
\end{array}\right.
$$

Now we obtain an integral representation of $p_{n}(x, y)$.

LEMMA 4.1. Let $d \chi$ be the normalized Haar measure on $\hat{\Gamma}$. Then,

$$
p_{n}(x, y)=\frac{1}{\ell\left(x_{0}, x\right)} \int_{\hat{\Gamma}}\left\langle P_{\chi}^{n} f_{y, \chi}, f_{x, \chi}\right\rangle_{\chi} d \chi .
$$

PROOF. Observe that

$$
\begin{aligned}
\left\langle P_{\chi}^{n} f_{y, \chi}, f_{x, \chi}\right\rangle_{\chi} & =\sum_{z \in \mathcal{F}}\left(\sum_{w \in V(G)} p_{n}(z, w) f_{y, \chi}(w)\right) \overline{f_{x, \chi}(z)} \ell\left(x_{0}, z\right) \\
& =\sum_{w \in V(G)} p_{n}(x, w) f_{y, \chi}(w) \ell\left(x_{0}, x\right) \\
& =\sum_{w \in \mathcal{F}} \sum_{\sigma \in \Gamma} p_{n}(x, \sigma w) f_{y, \chi}(\sigma w) \ell\left(x_{0}, x\right) \\
& =\sum_{\sigma \in \Gamma} p_{n}(x, \sigma y) \chi(\sigma) \alpha(\sigma) \ell\left(x_{0}, x\right) .
\end{aligned}
$$

Then integrating over $\hat{\Gamma}$ both sides of the equation above, and noting the orthogonality relations of unitary characters, we obtain

$$
\begin{aligned}
\int_{\hat{\Gamma}}\left\langle P_{\chi}^{n} f_{y, \chi}, f_{x, \chi}\right\rangle_{\chi} d \chi & =\ell\left(x_{0}, x\right) \int_{\hat{\Gamma}} \sum_{\sigma \in \Gamma} p_{n}(x, \sigma y) \chi(\sigma) \alpha(\sigma) d \chi \\
& =\ell\left(x_{0}, x\right) p_{n}(x, y) .
\end{aligned}
$$

This is the desired integral representation. 
In the same way, we obtain the following integral representation of $p_{t}(x, y)$.

Lemma 4.2. Let $\Delta_{\chi}=P_{\chi}-I$. Then,

$$
p_{t}(x, y)=\frac{1}{\ell\left(x_{0}, x\right)} \int_{\hat{\Gamma}}\left\langle e^{t \Delta_{\chi}} f_{y, \chi}, f_{x, \chi}\right\rangle_{\chi} d \chi .
$$

Now we recall the Laplace method. (See [2] for instance.)

THEOREM 4.3 (The Laplace method). Let $K$ be a compact set of $\boldsymbol{R}^{k}$, and let $f$ and $g$ be real-valued continuous functions on $K$. Suppose that $f$ attains the unique maximum at $x_{0}$ in the interior of $K, f$ is of $C^{2}$-class in a neighborhood of $x_{0}$ and the Hessian of $f$ at $x_{0}$ is negative definite. Then

$$
\int_{K} e^{n f(x)} g(x) d x \sim \frac{g\left(x_{0}\right)}{\left|\operatorname{det} \operatorname{Hess} f\left(x_{0}\right)\right|^{1 / 2}}\left(\frac{2 \pi}{n}\right)^{k / 2} e^{n f\left(x_{0}\right)}
$$

as $n \rightarrow \infty$. If, in addition, $|f|$ also attains the unique maximum at $x_{0}$, then

as $n \rightarrow \infty$.

$$
\int_{K} f(x)^{n} g(x) d x \sim \frac{g\left(x_{0}\right)}{\left|\operatorname{det} \operatorname{Hess} f\left(x_{0}\right)\right|^{1 / 2}}\left(\frac{2 \pi f\left(x_{0}\right)}{n}\right)^{k / 2} f\left(x_{0}\right)^{n}
$$

Now we are in a position to prove the theorems stated in Section 1.

Proof of Theorems 1.1 AND 1.3. We enumerated the eigenvalues of $P_{\chi}$ by $\left\{\lambda_{i}(\chi)\right\}_{i=1}^{N}$, and now let $\left\{\varphi_{\chi}, i\right\}_{i=1}^{N}$ be the corresponding normalized eigenfunctions with respect to $\|\cdot\|_{\chi}$. We assumed that $G$ is non-bipartite and hence, from Proposition 3.4, we get

$$
\max \left\{\left|\lambda_{i}(\chi)\right| \mid 2 \leq i \leq N \text { and } \chi \in \hat{\Gamma}\right\}<\lambda_{1}(\mathbf{1}) .
$$

By the spectral decomposition of $P_{\chi}$, we have

$$
P_{\chi}^{n}=\sum_{i=1}^{N} \lambda_{i}^{n}(\chi)\left\langle\cdot, \varphi_{\chi, i}\right\rangle_{\chi} \cdot \varphi_{\chi, i}
$$

and so by (4.9) together with Lemma 4.1

$$
\begin{aligned}
p_{n}(x, y) & =\frac{1}{\ell\left(x_{0}, x\right)} \sum_{i=1}^{N} \int_{\hat{\Gamma}} \lambda_{i}(\chi)^{n}\left\langle f_{y, \chi}, \varphi_{\chi}, i\right\rangle_{\chi}\left\langle\varphi_{\chi}, i, f_{x, \chi}\right\rangle_{\chi} d \chi \\
& \sim \frac{1}{\ell\left(x_{0}, x\right)} \int_{\hat{\Gamma}} \lambda_{1}(\chi)^{n}\left\langle f_{y, \chi}, \varphi_{\chi}, 1\right\rangle_{\chi}\left\langle\varphi_{\chi}, 1, f_{x, \chi}\right\rangle_{\chi} d \chi
\end{aligned}
$$

as $n \rightarrow \infty$.

Now, we take an orthonormal basis $\omega_{1}, \ldots, \omega_{k}$ of $T_{1} \hat{\Gamma}$ with respect to $\|\cdot\|_{q}$. Putting $\omega=x_{1} \omega_{1}+\cdots+x_{k} \omega_{k}$, we have a local coordinate system $\left(x_{1}, \ldots, x_{k}\right)$ of a neighborhood of the trivial character. In this coordinate system, since $d \chi$ is the normalized Haar measure, we get

$$
d \chi=d \chi_{\omega}=\frac{1}{\operatorname{Vol}_{q}(\hat{\Gamma})} d x_{1} \ldots d x_{k}
$$


Applying the Laplace method to (4.11), we obtain

$$
p_{n}(x, y) \sim \frac{1}{\ell\left(x_{0}, x\right)} \frac{\left\langle f_{y, \mathbf{1}}, \varphi_{\mathbf{1}, 1}\right\rangle_{\mathbf{1}}\left\langle\varphi_{\mathbf{1}, 1}, f_{x, \mathbf{1}}\right\rangle_{\mathbf{1}}}{\left|\operatorname{det}\left(\operatorname{Hess}_{\chi=1} \lambda_{1}\right)\right|^{1 / 2}}\left(\frac{2 \pi \lambda_{1}(\mathbf{1})}{n}\right)^{k / 2} \frac{\lambda_{1}(\mathbf{1})^{n}}{\operatorname{Vol}_{q}(\hat{\Gamma})} .
$$

We now consider $\varphi_{0} \in \ell^{2}(V(M))$ in (3.24) such that $\left\|\varphi_{0}\right\|_{0}=1$. Since $\varphi_{\mathbf{1}, 1}=U \varphi_{0}$ and $f_{x, 1}=U\left(\alpha\left(x, x_{0}\right) \delta_{\pi(x)}\right)$, we have

$$
\begin{aligned}
& \frac{1}{\ell\left(x_{0}, x\right)}\left\langle f_{y, \mathbf{1}}, \varphi_{\mathbf{1}, 1}\right\rangle_{\mathbf{1}}\left\langle\varphi_{\mathbf{1}, 1}, f_{x, \mathbf{1}}\right\rangle_{\mathbf{1}} \\
& \quad=\frac{1}{\ell\left(x_{0}, x\right)}\left\langle U\left(\alpha\left(y, x_{0}\right) \delta_{\pi(y)}\right), U \varphi_{0}\right\rangle_{\mathbf{1}} \cdot\left\langle U \varphi_{0}, U\left(\alpha\left(x, x_{0}\right) \delta_{\pi(x)}\right)\right\rangle_{\mathbf{1}} \\
& \quad=\frac{1}{\ell\left(x_{0}, x\right)}\left\langle\alpha\left(y, x_{0}\right) \delta_{\pi(y)}, \varphi_{0}\right\rangle_{0} \cdot\left\langle\varphi_{0}, \alpha\left(x, x_{0}\right) \delta_{\pi(x)}\right\rangle_{0} \\
& \quad=\ell(x, y)^{1 / 2} \varphi_{0}(\pi(x)) \varphi_{0}(\pi(y))
\end{aligned}
$$

Since $\left\{\omega_{i}\right\}_{i=1}^{k}$ is an orthonormal basis with respect to $\|\cdot\|_{q}$, we obtain $\left|\operatorname{det} \operatorname{Hess}_{\chi=1} \lambda_{1}\right|=$ $\left(8 \pi^{2}\right)^{k}$ by Lemma 3.5. Consequently, we get

$$
p_{n}(x, y) \sim \frac{\ell(x, y)^{1 / 2} \varphi_{0}(\pi(x)) \varphi_{0}(\pi(y))}{\operatorname{Vol}_{q}(\hat{\Gamma})} \frac{\lambda_{1}(\mathbf{1})^{n+k / 2}}{(4 \pi n)^{k / 2}}
$$

as $n \rightarrow \infty$.

For continuous time random walks, using Lemma 4.2, we obtain the conclusion in the same manner as above.

\section{REFERENCES}

[ 1 ] N. BiggS, Algebraic potential theory on graphs, Bull. London Math. Soc. 29 (1997), 641-682.

[ 2 ] N. Bleistein and R. A. Handelsman, Asymptotic expansions of integrals, Dover, New York, 1975.

[ 3 ] Y. GuivarC'H, Application d'un theoreme limite local a la transience et a la recurrence de marches de Markov, Theorie du potentiel (Orsay, 1983), 301-332, Lecture Notes in Math. 1096, Springer, Berlin, 1984.

[ 4 ] J. JACOD, Théorème de renouvellement et classification pour les chaînes semi-markoviennes, Ann. Inst. H. Poincaré Sect. B (N.S.) 7 (1971), 83-129.

[ 5 ] M. Kotani, T. Shirai AND T. SunAda, Asymptotic behavior of the transition probability of a random walk on an infinite graph, J. Funct. Anal. 159 (1998), 664-689.

[ 6] M. KotAni AND T. SunADA, Jacobian tori associated with a finite graph and its abelian covering graphs, Adv. in Appl. Math. 24 (2000), 89-110.

[ 7 ] M. Kotani and T. Sunada, Albanese maps and off diagonal long time asymptotics for the heat kernel, Comm. Math. Phys. 209 (2000), 633-670.

[ 8 ] A. KRÁMLi And D. SzÁsz, Random walks with internal degrees of freedom, Z. Wahrsch. Verw. Gebiete 63 (1983), 85-95.

[ 9 ] F. SPITZER, Principles of random walk, D. Van. Nostrand, Princeton, N. J.-Toronto-London, 1964.

[10] W. WoEss, Random walks on infinite graphs and groups, Cambridge Tracts in Mathematics 138, Cambridge University Press, Cambridge, 2000. 
DEPARTMENT OF COMPUTATIONAL SCIENCE

KANAZAWA UNIVERSITY

KAKUMA-MACHI, KANAZAWA

ISHIKAWA 920-1192

JAPAN

E-mail address: shirai@kenroku.kanazawa-u.ac.jp 